Casos Clínicos

Arch. Esp. Urol., 60, 5 (580-582), 2007

\section{VARICOCELE INTRATESTICULAR ASOCIADO A ATROFIA GLANDULAR Y A ALTERACIONES ECOGRÁFICAS PARENQUIMATOSAS.}

\author{
Antonio Javier Márquez Moreno, Emilio Julve Villalta' y \\ José Ruíz Escalante.
}

Servicios de Radiodiagnóstico y de Urología'. Hospital Clínico. Málaga. España.

Resumen.- OBJETIVO: Describir un nuevo caso de varicocele intratesticular (patología infrecuente - $2 \%$ de los casos-) con una serie de peculiaridades ecográficas: atrofia testicular y alteraciones parenquimatosas asociadas, en un paciente de 24 años, con dolor testicular izquierdo.

MÉTODOS: Se realizó ecografía doppler color, y estudio ecográfico urológico, así como TAC abdominopélvico.

RESULTADOS: En el estudio ecográfico se apreció un severo varicocele izquierdo, de tipo mixto, con componente habitual extratesticular y con vasos dilatados subcapsulares y paramediastínicos intratesticulares, con flujos turbulentos que aumentaban con maniobras de Valsalva, así como una alteración de la ecogenicidad parenquimatosa, con áreas hipoecoicas e hipovasculares y disminución del tamaño testicular.
DISCUSIÓN/CONCLUSIONES: El varicocele intratesticular es una patología infrecuente que muestra predilección por el testículo izquierdo, asociándose frecuentemente a varicocele extratesticular. La presencia de atrofia testicular y las alteraciones parenquimatosas asociadas son hechos raramente descritos en la literatura. Clínicamente muestran aspecto solapables con las forma extratesticulares.

Palabras clave: Testículo. Varicocele intratesticular. Atrofia. Doppler-color.

Summary.- OBJECTIVE: To report one case of intratesticular varicocele, infrequent pathology (2\% of the cases), with a series of ultrasound peculiarities: testicular atrophy and associated parenchymal abnormalities, in a 24-year-old patient with left testicular pain.

METHODS: Ultrasound showed severe left varicocele, mixed type, with the regular extratesticular component and dilated subcapsular and mediastinum vessels, with turbulent flows increasing with Valsalva's manoeuvre, as well as an alteration of the ultrasound pattern of the parenchyma, with hypoechoic and hypovascular areas and diminished testicular size.

DISCUSSION/CONCLUSIONS: Intratesticular varicocele is a rare pathology more frequent in the left testicle, which is frequently associated with extratesticular varicocele. The presence of testicular atrophy and associated parenchymal abnormalities has been rarely described in the literature. Clinically they show features overlapping the extratesticular types.

Keywords: Testicle. Intratesticular varicocele. Atrophy. Color Doppler ultrasound.

\title{
INTRODUCCIÓN
}

El varicocele es una patología frecuente que suele afectar a la porción extratesticular del plexo venoso, sin embargo, en un $2 \%$ de los casos se observa una afectación venosa intratesticular, relacionada, en la mayoría de los casos a un componente de afectación extratesticular, pudiendo asociarse a situaciones clínicas variadas o ser asintomáticos (1 y 2).

\section{CASO CLÍNICO}

Antonio Javier Márquez Moreno

Teseo, 5 3-N.

290 10. Málaga. (España).

nuriaantonio@terra.es

Trabajo recibido: 29 de septiembre 2006

Paciente de 24 años, sin antecedentes de interés, que es remitido por ligero dolor testicular izquierdo de larga evolución, sin otros datos de interés. Se realiza ecogra- 


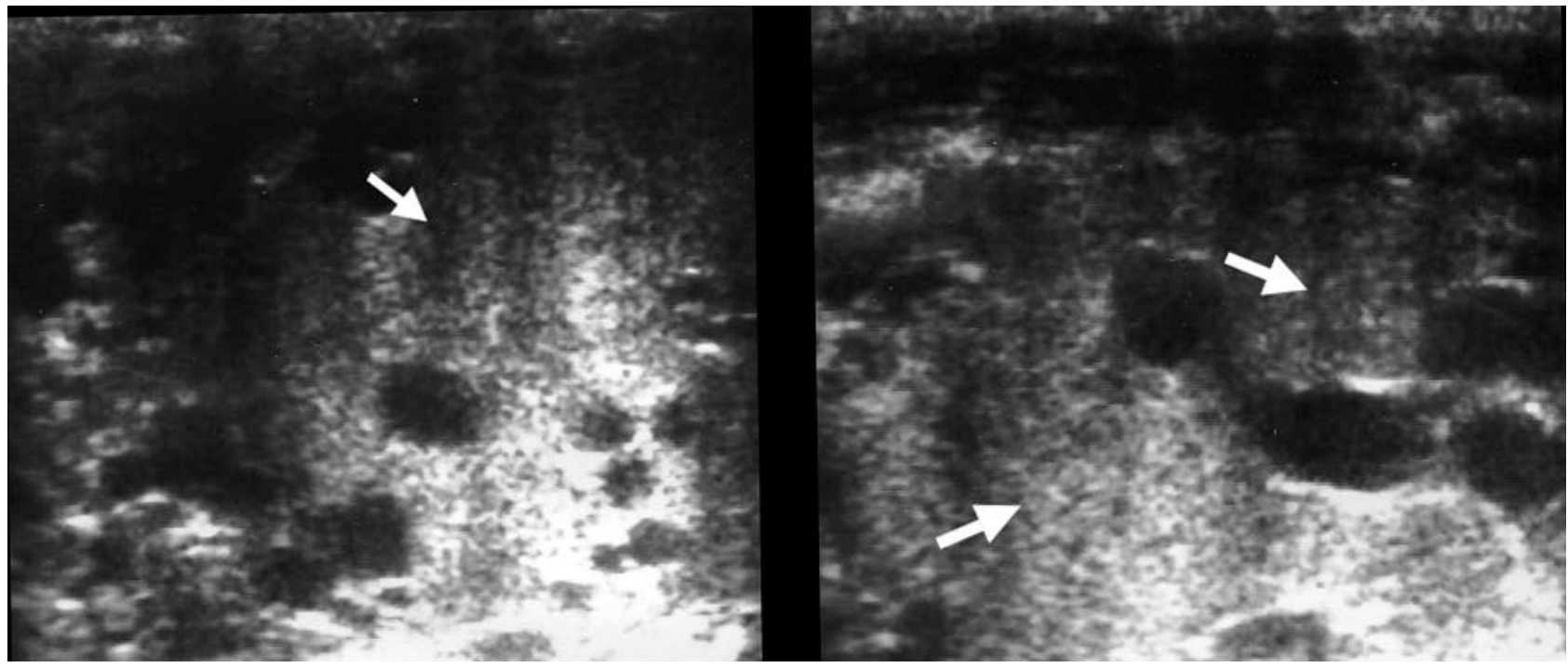

FIGURA 1. Ecografía: dilataciones vasculares extra e intratesticulares, con escaso parénquima residual, de aspecto heterogéneo (flechas).

fía doppler testicular mostrando teste derecho sin alteraciones. En el lado izquierdo se observa una marcada disminución del tamaño testicular, con ecoestructura hipoecoica parcheada, sin masas evidentes y una severa dilatación del plexo venoso, con vasos tortuosos y un componente intratesticular importante, en localización subcapsular y en el mediastino testicular (Figuras 1 y 2). Se aprecia disminución del número de vasos en las zonas hipoecoicas (eco-dopler color, "angio-power" -modo energía-) y un flujo espontáneo en los vasos varicosos, que aumenta con maniobras de Valsalva. El resto del estudio ecográfico vesicoprostático y renal, así como la TAC abdominopélvica son normales.

\section{COMENTARIO}

El varicocele extratesticular es una patología frecuente que afecta a alrededor de un $20 \%$ de la población, y que está originada por una anormal dilatación de las venas del plexo pampiniforme, cuyo diámetro normal, es, generalmente, y en las venas de mayor calibre (vena principal de drenaje), de unos $2 \mathrm{~mm}$. En un $2 \%$ de los casos la dilatación venosa afecta a los vasos intratesticulares, y generalmente $(80 \%)$ se asocia a un varicocele extratesticular. Al igual que en la literatura consultada, nuestro caso muestra predominio izquierdo $147 \%$ de los casos afectan al lado izquierdo, $13 \%$ al derecho y en un $20 \%$ la patología es bilateral), y los vasos intratesticulares se localizan subcapsulares y paramediastínicos $160 \%$ y $4 \%$ respectivamente en la literatura consultada). El flujo en los vasos se registra de forma espontánea y aumenta con maniobra de Valsalva, y la morfología de los vasos venosos dilatados es mixta (tubular y oval) (3 y 4). Vasilios y cols. (2006) describen unos 34 casos de varicocele intratesticulares registrados en la literatura
(2). Sin embargo, la presencia de atrofia testicular asociada sólo ha sido descrita por Ho y cols. y Conti y cols. (ambos en el 2005) (5 y 6). Además, nosotros hemos observado alteraciones parenquimatosas ecográficas, en forma de una ecogenicidad testicular alterada, parcheada, y con foco hipoecoicos geográficos, sin masas evidentes, posiblemente secundarias a alteraciones vasculares locales.

Al igual que en los varicoceles extratesticulares, se han descrito situaciones clínicas (que se solapan en ambos tipos de varicoceles, lo cual no es raro ya que los dos

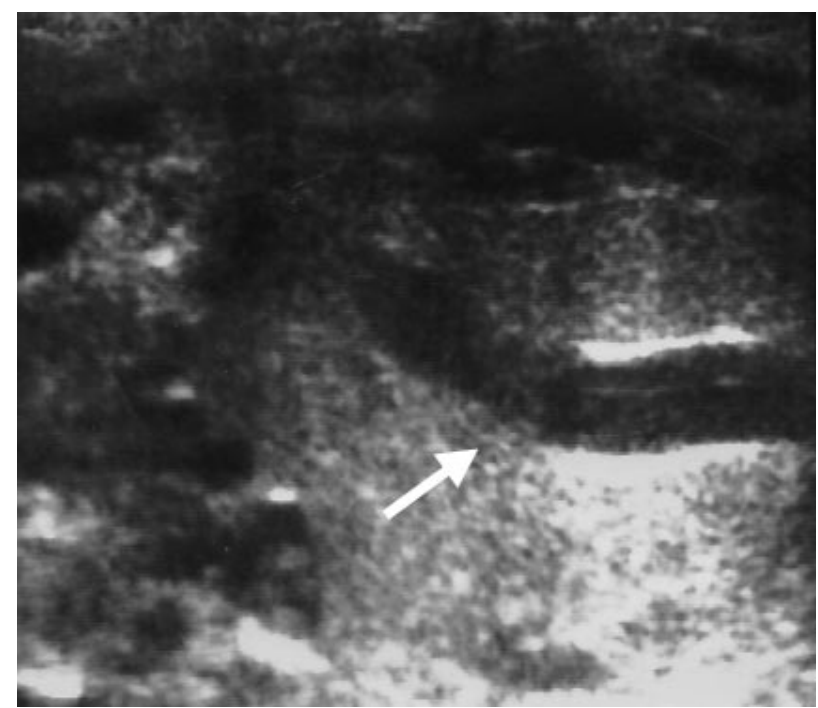

FIGURA 2. Ecografía. Estructura vascular intratesticular marcadamente dilatada. 
coexisten en un $85 \%$ de los casos) asociadas al varicocele intratesticular: infertilidad, dolor testicular o masa testicular, no faltando los casos asintomáticos $(5,6,7)$.

En los casos en los que se requiere tratamiento, se puede realizar una ligadura de la vena espermática, habiéndose descrito la embolización percutánea como alternativa, por algunos autores $(8,9)$.

\section{BIBLIOGRAFÍA y LECTURAS RECOMENDADAS (*lectura de interés $y$ ** lectura fundamental)}

**1. PASCUAL, C.; FERNÁNDEZ, I.; RODRÍGUEZ, N. y cols.: "Ultrasound diagnosis of intratesticular varicocele". Arch. Esp., 58: 963; 2005.

**2. VASILIOS, S.; CHARALAMPOS, L.; ELIAS, P. y cols.: "Ultrasound findings of an intratesticular varicocele. Report of a new case and review of the literatura". Int. Urol. Nephrol., 38: 115, 2006.

3. BROWNE, R.; GEOGHEAN, T.; AHMED, I. y cols.: "Intratesticular varicocele". Australasia Radiol., 49: 333, 2005

4. KESSLER, A.; MEIRSDORF, S.; GRAIF, M. y cols.: "Intratesticular varicocele: gray scale and color Doppler sonographic appearance”. J. Ultrasound Med., 2: $1711,2005$.

**5. HO, K.; McATEER, E.; YOUNG, M.: "Loss of testicular volume associated with intratesticular varicocele". Int. J. Urol., 12: 422; 2005.

6. CONTI, E.; FASOLO, P.; SEBASTIÁN, G. y cols.: "Color Doppler sonography in the intratesticular varicocele". Arch. Ital. Urol. Androl., 77: 63, 2005.

*7. SÁNCHEZ, A.; VILLOR, R.; PAMPLONA, M.: "Radiological diagnosis: scrotal ultrasound and Doppler ultrasound in the diagnosis of male infertility". Arch. Esp. Urol., 57: 905; 2004.

*8. DIAMOND, D.; ROTH, J.; CILENTO, B. y cols.: "Intratesticular varicocele in adolescents: a reversible anechoic lesion of the testis". J. Urol., 171: 381, 2004.

*9. DEMIRBAS, M.; ELLERGEZEM, A.; BILEN, C. y cols.: "Intratesticular varicocele treated with percutaneous embolization". Urology, 58: 1058, 2001.
Casos Clínicos

Arch. Esp. Urol., 60, 5 (582-585), 2007

\section{SEMINOMA Y TERATOCARCINOMA: ¿PRESENTACIÓN SINCRÓNICA MONOTESTICULAR COMO NÓDULOS INDEPENDIENTES CON DISTINTAS HISTOLOGIAS? CARACTERES ECOGRÁFICOS.}

\author{
Irene García Bocanegra, Antonio Javier Márquez \\ Moreno, Emilio Julve Villalta', Lidia Pérez Villa², José \\ Ruíz Escalante y Alfredo Blanes Berenguel².
}

Servicios de Radiodiagnóstico, Urología' y Anatomía Patológica². Hospital Clínico. Málaga. España.

Resumen.- OBJETIVO: Describir las características ecográficas, el patrón de vascularización (eco-doppler color) y la posible histogénesis de un caso de presentación sincrónica monotesticular de tumor seminomatoso y teratocarcinoma como nódulos tumorales independientes e histológicamente distintos, en un paciente de 19 años, con una masa testicular de 8 meses de evolución.

MÉTODOS: Se realizó estudio convencional ecográfico, eco doppler color y angio-doppler con ecógrafo de alta resolución, analizando los flujos vasculares. Tras la resección del tumor se correlacionaron las secciones macroscópicas e histológicas con los planos ecográficos realizados.

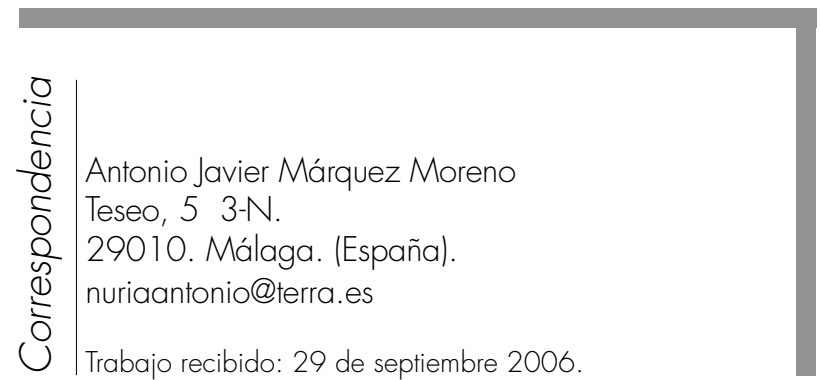

